\title{
RELATIVE COHOMOLOGY AND PROJECTIVE TWISTOR DIAGRAMS
}

\author{
S. A. HUGGETT AND M. A. SINGER
}

\begin{abstract}
The use of relative cohomology in the investigation of functionals on tensor products of twistor cohomology groups is considered and yields a significant reduction in the problem of looking for contours for the evaluation of (projective) twistor diagrams. The method is applied to some simple twistor diagrams and is used to show that the standard twistor kernel for the first order massless scalar $\phi^{4}$ vertex admits a (cohomological) contour for only one of the physical channels. A new kernel is constructed for the $\phi^{4}$ vertex which admits contours for all channels.
\end{abstract}

\section{INTRODUCTION}

Since the discovery that the twistor description of massless fields is best understood in terms of the analytic cohomology of twistor space [3, 9, 13], the question of how to interpret twistor diagrams $[6,16]$ in a cohomologically satisfactory way has attracted considerable interest $[4,5,8]$. The introduction of such diagrams is motivated by the desire to extend the twistor description of massless fields so as to include their basic (quantum field theoretic) interactions.

A Feynman diagram specifies a kernel which defines, by substitution into an appropriate integral, a functional on some tensor product of Hilbert spaces. Similarly, a twistor diagram specifies a holomorphic kernel $h$ which is to define a functional on some tensor product of analytic cohomology groups. In fact, one generally expects on physical grounds to associate a (finite) family of functionals to any one kernel, since there are generally many physically significant 'channels' for any one Feynman diagram. These functionals arise by performing a 'contour integral' of the product of the kernel with appropriate cohomology classes representing massless fields. This contour integral is better thought of as pairing with a (compact) homology class defined on a space which is (typically) the complement of an analytic subvariety-the singularity set of $h$-in a product of open subsets of projective twistor space.

Received by the editors December 10,1988. The main ideas in this article were presented on the 11 th of July 1988 to the London Mathematical Society Symposium on Spinors, Twistors, and Complex Structures in General Relativity, in Durham.

1980 Mathematics Subject Classification (1985 Revision). Primary 81C30; Secondary 32L25.

The first author was on leave from Plymouth Polytechnic during 1986-1987, and the second author was a Junior Research Fellow, Merton College, Oxford. 
In $\S 3$ of this paper, we formulate, in a rather general setting, a significant simplification of the problem of finding all such homology classes and apply it, in $\S 4$, in some simple examples. What appears to be new in our approach is the thoroughgoing use of Serre duality and analytic relative cohomology: the relevant facts about these subjects are gathered in $\S 2$; a description in terms of differential forms appears in Appendix A.

The examples discussed in $\S 4$ comprise two twistor-theoretic representations of the scalar product between massless fields and a discussion of the first-order massless scalar $\phi^{4}$ vertex. With regard to the former, we reproduce already known results; our results concerning the latter, however, are new. Indeed, our method shows that for the standard twistor-theoretic kernel for the $\phi^{4}$ vertex, there is a homology class (contour) for only one of the three physical channels. As we shall see, however, there is an alternative kernel for the same vertex which yields all physical channels. Appendix B is devoted to a brief derivation of this kernel from space-time considerations.

\section{ANALYTIC RELATIVE COHOMOLOGY}

We shall outline here the facts about relative sheaf cohomology that will be required in $\S 3$. Most of the abstract theory can be found in standard books on sheaf theory such as [10]. Analytic relative sheaf cohomology can be represented concretely in terms of differential forms-an outline of this appears in Appendix A.

2.1. Relative cohomology and compact relative cohomology. Let $X$ be a complex manifold of complex dimension $n$; let $B \rightarrow X$ be some holomorphic vector bundle; and denote by $\Omega^{p}(B) \quad(0 \leq p \leq n)$ the bundle of $B$-valued $p$-forms on $X$.

Let $F$ be a closed subset of $X$ and set $U=X-F$. We can then introduce the relative sheaf cohomology groups of $(X, U)$ with coefficients in $\Omega^{p}(B)$ which are written in any of the following ways:

$$
\begin{array}{lr}
H^{p, q}(X, U ; B), & H^{q}\left(X, U ; \Omega^{p}(B)\right), \\
H_{F}^{p, q}(X ; B), & H_{F}^{q}\left(X ; \Omega^{p}(B)\right) .
\end{array}
$$

These groups are generalisations of the 'absolute' sheaf cohomology groups since

$$
H^{p, q}(X, \varnothing ; B) \equiv H_{X}^{p, q}(X ; B)=H^{p, q}(X ; B) ;
$$

they depend only upon $F$ and its immediate neighbourhood in $X$; and they are linked to the sheaf cohomology of $X$ and $U$ by means of the relative cohomology exact sequence

$$
\rightarrow H_{F}^{p, q}(X ; B) \rightarrow H^{p, q}(X ; B) \rightarrow H^{p, q}(U ; B) \rightarrow H_{F}^{p, q+1}(X ; B) \rightarrow .
$$

We also need the 'dual theory', viz. the compact relative sheaf cohomology of $(X, U)$ with coefficients in $\Omega^{p}(B)$, written

$$
H_{c}^{p, q}(X, U ; B) \text { or } H_{c}^{q}\left(X, U ; \Omega^{p}(B)\right) .
$$


This group reduces to the compact sheaf cohomology of $X$ when $U=\varnothing$, depends only upon $F$ and its immediate neighbourhood in $X$, and is linked to the compact cohomology of $X$ and $U$ by the following exact sequence:

(2) $\rightarrow H_{c}^{p, q}(U ; B) \rightarrow H_{c}^{p, q}(X ; B) \rightarrow H_{c}^{p, q}(X, U ; B) \rightarrow H_{c}^{p, q+1}(U ; B) \rightarrow$.

We referred to compact relative sheaf cohomology as the 'dual theory' for the following reason. Let $\check{B}$ denote the bundle dual to $B$. Then there is a commutative diagram

$$
\begin{aligned}
& \rightarrow H^{p, q}(X ; B) \quad \rightarrow H^{p, q}(U ; B) \quad \rightarrow H^{p, q+1}(X, U ; B)
\end{aligned}
$$

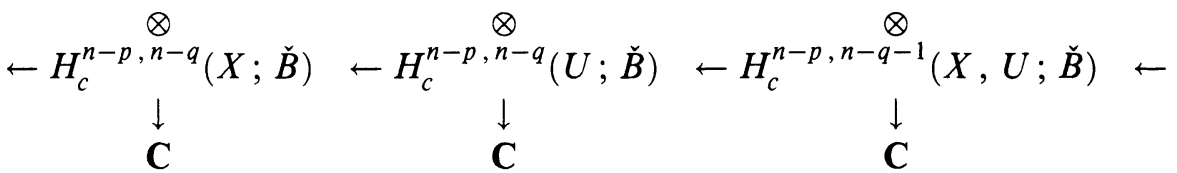

in which the first two vertical pairings are the standard ones induced by

$$
(\alpha, \beta) \mapsto \int_{X} \alpha \wedge \beta
$$

(in terms of a differential-form description of the relevant sheaf cohomology groups). We shall denote the pairing of

$$
\xi \in H^{p, q}(X, U ; B) \text { and } \eta \in H_{c}^{n-p, n-q}(X, U ; \check{B})
$$

by

$$
(\xi, \eta) \mapsto(\xi \cup \eta)[X] .
$$

In [14], Serre showed that when one endows the cohomology groups

$$
H^{p, q}(X, B)
$$

and

$$
H_{c}^{n-p, n-q}(X, \check{B})
$$

with certain natural topologies, (3) identifies each as the topological dual of the other, subject to certain closed-range hypotheses on the $\bar{\partial}$-operator. It is a consequence of the work of Laufer [11] that any continuous linear functional on $(5)$ is of the form

$$
\alpha \mapsto \int_{X} \alpha \wedge \beta
$$

for some $\beta$ in (6)-but that $\beta$ need not be unique. When combined with the five-lemma in the above commutative diagram, this fact implies the following

Proposition 2.1. Every continuous linear functional on $H^{p, q}(X, U ; B)$ is of the form

$$
\xi \mapsto(\xi \cup \eta)[X],
$$

where $\eta$ is some element of $H_{c}^{n-p, n-q}(X, U ; \check{B})$. 
If $p=n$ and $B$ is canonically trivial, there is a distinguished subclass of functionals arising from the natural map

$$
H_{n+q}(X, U ; \mathbf{C}) \equiv H_{c}^{n-q}(X, U ; \mathbf{C}) \rightarrow H_{c}^{0, n-q}(X, U)
$$

induced by the embedding of the constant sheaf $\mathbf{C}$ in $\mathscr{O}$. One should think of these as arising by 'contour integration' or better as pairing a relative sheaf cohomology class with a relative homology class.

Consider now the further special case in which $F$ is a closed submanifold of $X$ with orientable normal bundle. Then if the real codimension of $F$ in $X$ is $k$, we have, for each integer $q \geq k-n$, the Thom isomorphism

$$
H_{n+q-k}(F) \rightarrow H_{n+q}(X, U)
$$

induced by mapping a cycle $c$ in $F$ into the relative cycle obtained by replacing each point of $c$ by a small $k$-dimensional disc normal to $F$. The inverse map is given by intersection of transverse relative cycles with $F$.

Combining (7) and (8) we arrive at the result that will be of central importance in $\S 3$.

Theorem 2.2. Let $F$ be a closed submanifold of $X$ of real codimension $k$, with orientable normal bundle. Then for each integer $q \geq k-n$ there is a natural map from $H_{n+q-k}(F)$ into the space of continuous linear functionals on $H^{n, q}(X, U)$.

The combination of (2) and (8) yields the Leray exact sequence $[12,16]$ :

$$
\rightarrow H_{q+1-k}(F) \stackrel{\delta}{\rightarrow} H_{q}(U) \rightarrow H_{q}(X) \stackrel{\cap}{\rightarrow} H_{q-k}(F) \rightarrow \text {. }
$$

By the above description of the Thom isomorphism, the map denoted by $\cap$ carries a representative cycle (which is assumed to be transversal to $F$ ) to its intersection with $F$, while $\delta$ (Leray's 'cobord' map) fibres a cycle in $F$ with small normal $(k-1)$-spheres.

2.2. Products on relative cohomology groups. Let $X$ and $Y$ be complex manifolds, let $F \subset X$ and $G \subset Y$ be closed subsets, and let $B \rightarrow X$ and $C \rightarrow Y$ be holomorphic vector bundles. Denoting by $\pi_{X}$ and $\pi_{Y}$ the natural projections from $X \times Y$ onto $X$ and $Y$, we define

$$
B \times C=\pi_{X}^{*} B \otimes \pi_{Y}^{*} C
$$

on $X \times Y$. Then it is a consequence of standard abstract nonsense that there is a product, to be called the cross product in relative cohomology, such that

$$
H_{F}^{p}(X ; B) \otimes H_{G}^{q}(Y ; C) \rightarrow H_{F \times G}^{p+q}(X \times Y ; B \times C)
$$

(there is an explicit formula in Appendix A). Conversely, we have a Künneth formula:

$$
H_{F \times G}^{r}(X \times Y ; B \times C)=\bigoplus_{p+q=r} H_{F}^{p}(X ; B) \otimes H_{G}^{q}(Y ; C) .
$$


The cross product is natural with respect to the relative cohomology exact sequence (1): if $\alpha \in H_{F}^{p}(X ; B), \beta \in H_{G}^{q}(Y ; C)$, and $\alpha$ maps to zero in $H^{p}(X ; B)$, then $\alpha \times \beta$ maps to zero in

$$
H^{p+q}(X \times Y ; B \times C) ;
$$

a similar statement is of course true for $\beta$. In particular if $\alpha$ is the image of $\alpha_{0} \in H^{p-1}(X-F ; B)$ and $\beta$ is the image of $\beta_{0} \in H^{q-1}(Y-G ; C)$, then $\alpha \times \beta$ is in the image of the group

$$
H^{p+q-1}(X \times Y-F \times G ; B \times C) .
$$

In fact there is a well-defined class $\alpha_{0} \cdot \beta_{0}$ in this group, called the dot product of $\alpha_{0}$ and $\beta_{0}$, such that

$$
\alpha_{0} \cdot \beta_{0} \mapsto \alpha \times \beta
$$

under the connecting homomorphism. (Again, there is a formula in Appendix A.)

This characterisation of the dot product was communicated to us by R. J. Baston. It can also be defined directly in terms of Čech representatives or by use of the Mayer-Vietoris sequence (cf. $[4,5])$. We shall see in the next section the advantage of the present point of view.

\section{A GENERAL PROCEDURE}

Here we shall apply the material assembled in $\S 2$ to investigate the functionals on certain cohomology groups defined in open subsets of projective twistor space. Recall that nonprojective twistor space $\mathbf{T}$ is a complex vector space of dimension four with dual space $\mathbf{T}^{*}$. Then (projective) twistor space $\mathbf{P}$ is the projective space of $\mathbf{T}$, (projective) dual twistor space $\mathbf{P}^{*}$ is the projective space of $\mathbf{T}^{*}$, and (complexified, compactified) Minkowski space $\mathbf{M}$ appears as the Grassmannian of complex 2-planes in $\mathbf{T}$. The reader is referred to $[3,9,13]$ for further information and notation.

Throughout, we shall use the following notation: For each $j(j=1, \ldots, m)$, $P_{j}$ will stand for either $\mathbf{P}$ or $\mathbf{P}^{*}$, and $U_{j}$ will be some open subset of $P_{j}$ which is swept out by projective lines corresponding to points in some convex set $V_{j}$ in Minkowski space. This is sufficient to guarantee that the Penrose transform (cf. [3]) is an isomorphism between $H^{1}\left(U_{j} ; \mathscr{O}(-2 s-2)\right)$ and the space of holomorphic solutions on $V_{j}$ of the massless field equations of helicity $s$. We shall also write $F_{j}=P_{j}-U_{j}, L_{j}$ for a projective line contained in $F_{j}, \Pi=P_{1} \times \cdots \times P_{m}$, $F=F_{1} \times \cdots \times F_{m}$, and $\Lambda=L_{1} \times \cdots \times L_{m}$.

What we want to understand is the space of functionals on the tensor product

$$
H^{1}\left(U_{1} ; \mathscr{O}\left(r_{1}\right)\right) \otimes \cdots \otimes H^{1}\left(U_{m} ; \mathscr{O}\left(r_{m}\right)\right),
$$

where $r_{j}$ is a family of integers; in particular we are interested in those that are determined, according to the recipe in the Introduction, by a holomorphic kernel

$$
h \in H^{3 m, q}(\Pi-\Sigma ; \mathscr{O}(-\mathbf{r})) .
$$


In the last group we have used the abbreviation

$$
\mathscr{O}(-\mathbf{r})=\mathscr{O}\left(-r_{1}\right) \times \cdots \times \mathscr{O}\left(-r_{m}\right)
$$

(cf. $\S 2.2$ for the definition of the right-hand side), and $\Sigma$, the 'singularity set' of $h$, is typically some (possibly singular) analytic subvariety of $\Pi$.

Using the cup product to multiply an element of (13) by $h$ we obtain an element of

$$
H^{3 m, m+q}\left(U_{1} \times \cdots \times U_{m}-\Sigma\right) ;
$$

so applying (7) with $X=U_{1} \times \cdots \times U_{m}-\Sigma$ and $U=\varnothing$ we see that the functionals in which we are interested are given by homology classes in

$$
H_{4 m+q}\left(U_{1} \times \cdots \times U_{m}-\Sigma\right) \text {. }
$$

This group is, however, somewhat intractable-we shall see that by using relative cohomology its study can be much simplified.

For this, it is necessary to make some mild assumptions. First we demand that the problem is 'well-posed', viz. that

$$
F \not \subset \Sigma \text {. }
$$

We shall see below that there are no relevant functionals for ill-posed problems. In the examples of $\S 4$, moreover, (16) has a rather natural geometric meaning. In the rest of this section, we shall assume that the problem is well-posed unless the contrary is explicitly stated. Then under a further mild condition (cf. (25) below), the functionals defined by elements in (15) also arise from classes in $H_{m+q}(\Lambda-\Sigma)$. Now $\Lambda-\Sigma$ is much easier to deal with than the domain of (15): it is of much lower dimension $(2 \mathrm{~m}$ rather than $6 \mathrm{~m})$ and is typically just the complement of an analytic subvariety in the product of $m$ copies of the Riemann sphere.

3.1. Twistor relative cohomology classes. Our definition of $U_{j}$ guarantees that

$$
H^{k}\left(U_{j} ; \mathscr{O}\left(r_{j}\right)\right)=0 \text { if } k \geq 2
$$

and

$$
H^{0}\left(U_{j} ; \mathscr{O}\left(r_{j}\right)\right)=H^{0}\left(P_{j} ; \mathscr{O}\left(r_{j}\right)\right) .
$$

Note also that $F_{j}$ is homotopic to $L_{j}$.

Combining these with the standard theorems on the cohomology of $P_{j}$, viz.

$$
H^{k}\left(P_{j} ; \mathscr{O}\left(r_{j}\right)\right)=0 \text { if } 0<k<3,
$$

we obtain from the relative cohomology exact sequence (1) that

$$
H_{F_{j}}^{k}\left(P_{j} ; \mathscr{O}\left(r_{j}\right)\right)=0 \text { if } k=0,1
$$

and

$$
H^{1}\left(U_{j} ; \mathscr{O}\left(r_{j}\right)\right)=H_{F_{j}}^{2}\left(P_{j} ; \mathscr{O}\left(r_{j}\right)\right) \text {. }
$$

Using this isomorphism and the cross product (10), we obtain the following 
Proposition 3.1. The tensor product of cohomology groups (13) is canonically isomorphic to the relative cohomology group

$$
H_{F}^{2 m}(\Pi ; \mathscr{O}(\mathbf{r}))
$$

for all $m$ and $\mathbf{r}=\left(r_{1}, \ldots, r_{m}\right)$.

Proof. By the Künneth formula (11) for relative cohomology,

$$
H_{F}^{2 m}(\Pi ; \mathscr{O}(\mathbf{r}))=\bigoplus \bigotimes_{j=1}^{n} H_{F_{j}}^{q_{j}}\left(P_{j} ; \mathscr{O}\left(r_{j}\right)\right),
$$

where the direct sum is over all integers $q_{j}$ such that $q_{1}+\cdots+q_{m}=2 m$. In view of (18), (13) appears as a summand with $q_{j}=2$ for all $j$. For any other allowable collection of integers $q_{j}$, we must have some $q_{j}<2$. But the corresponding summand vanishes by (17).

Note that if we used the dot product (12) to multiply together the groups in (13) then we would get into the group

$$
H^{2 m-1}(\Pi-F ; \mathscr{O}(\mathbf{r})) .
$$

Because the cross product is compatible with the relative cohomology exact sequence, the proposition that we have just proved says that this dot product is injective; indeed its image is mapped injectively by the connecting homomorphism into (19). In particular, there is no 'loss of information' in using the dot product. From the point of view of the other definitions of this product, that is not so obvious.

3.2. Functionals defined by a holomorphic kernel. By Proposition 3.1 and 2.1, the space of continuous functionals on (13) is given by the compact relative cohomology group

$$
H_{c}^{3 m, m}(\Pi, \Pi-F ; \mathscr{O}(-\mathbf{r})) .
$$

It remains to show how a holomorphic kernel $h$ as in (14) picks out a preferred class of functionals in (21). Note first that there is a natural map

$$
H_{c}^{3 m, m}(\Pi-\Sigma, \Pi-\Sigma \cup F ; \mathscr{O}(-\mathbf{r})) \stackrel{l}{\rightarrow} H_{c}^{3 m, m}(\Pi, \Pi-F ; \mathscr{O}(-\mathbf{r}))
$$

induced by the open inclusion $\Pi-\Sigma \rightarrow \Pi$. Thus it is sufficient to show how the kernel $h$ picks out a family of elements in the space on the left-hand side of (22). But that is very easy, because

$$
H^{3 m, q}(\Pi-\Sigma ; \mathscr{O}(-\mathbf{r})) \otimes H_{c}^{0, m-q}(\Pi-\Sigma, \Pi-\Sigma \cup F)
$$

is mapped by the cup product into the left-hand side of (22).

The functionals in which we are interested are those in the image of (7), with

$$
X=\Pi-\Sigma, \quad U=\Pi-\Sigma \cup F .
$$

That is, they are of the form

$$
\imath[h \cup \alpha],
$$


where $\alpha$ lies in the image of

$$
H_{5 m+q}(\Pi-\Sigma, \Pi-\Sigma \cup F) \rightarrow H_{c}^{0, m-q}(\Pi-\Sigma, \Pi-\Sigma \cup F) .
$$

We shall refer to such a functional as being associated to or determined by the kernel $h$.

It is here that condition (16), that the problem be well-posed, enters. For if this condition is violated, we have $\Sigma \cup F=\Sigma$ and the relative cohomology groups of the pair $(\Pi-\Sigma, \Pi-\Sigma \cup F)$ are all zero. Thus the above arguments show that if the problem is ill-posed, any associated functional is zero.

Returning to the more interesting situation in which (16) is satisfied, let us first consider the important special case in which $F_{j}=L_{j}$ for each $j$. Here we have

\section{Theorem 3.2. The functionals on}

$$
H^{1}\left(P_{1}-L_{1} ; \mathscr{O}\left(r_{1}\right)\right) \otimes \cdots \otimes H^{1}\left(P_{m}-L_{m} ; \mathscr{O}\left(r_{m}\right)\right)
$$

associated to the kernel $h \in H^{3 m, q}(\Pi-\Sigma ; \mathscr{O}(-\mathbf{r}))$ are given by the elements of the homology group

$$
H_{m+q}(\Lambda-\Sigma)
$$

Proof. This is immediate from Theorem 2.2 since $\Lambda-\Sigma$ is a closed submanifold of $\Pi-\Sigma$ of real codimension $4 m$ whose normal bundle is orientable.

Note that we have used the rather imprecise phrase 'given by' in the statement of this theorem because, although every functional associated to $h$ comes from a homology class in (24), we do not (yet) know that every such nonzero homology class yields a nonzero functional.

Let us now turn to the more general situation in which $F_{j} \neq L_{j}$. We mentioned above that $F$ and $\Lambda$ are homotopic spaces; we shall make the further assumption that

$$
(\Pi-\Sigma, \Pi-\Sigma \cup F) \text { is homotopic to }(\Pi-\Sigma, \Pi-\Sigma \cup \Lambda) \text {; }
$$

one expects this to be satisfied if $F$ is not too large a neighbourhood of $\Lambda$ and $\Lambda$ is 'generically placed' with respect to $\Sigma$.

If $(25)$ holds, we have

$$
H_{*}(\Pi-\Sigma, \Pi-\Sigma \cup F)=H_{*}(\Pi-\Sigma, \Pi-\Sigma \cup \Lambda)
$$

and may deduce the following extension of Theorem 3.2.

Theorem 3.3. Let the closed subsets $F_{j} \subset P_{j}$ and $\Sigma$ satisfy (25). Then the functionals on

$$
H^{1}\left(U_{1}, \mathscr{O}\left(r_{1}\right)\right) \otimes \cdots \otimes H^{1}\left(U_{m}, \mathscr{O}\left(r_{m}\right)\right)
$$

associated to $h \in H^{3 m, q}(\Pi-\Sigma ; \mathscr{O}(-\mathbf{r}))$ are given by the elements of the homology group $H_{m+q}(\Lambda-\Sigma)$. 


\section{Applications}

In this section we give four examples of the application of our general procedure to the description of functionals associated to the kernels of the simplest projective twistor diagrams. In the first examples we are concerned with two different forms of the so-called scalar product diagram and the second two deal with the first-order massless $\phi^{4}$ vertex.

The notation we use is as in the previous sections of this paper. In particular, $P_{1}, \ldots, P_{4}$ are copies of projective twistor space or its dual and for each $j$, $L_{j}$ is a projective line in $P_{j}$, representing the point $v_{j}$ in Minkowski space. In addition, however, we shall need some notations for other twistor-theoretic pieces of furniture-for a fuller account, see $[9,13]$. We use $W, Y$ or $W_{\alpha}$, $Y_{\alpha}$ for general points of dual twistor space and $X, Z$ or $X^{\alpha}, Z^{\alpha}$ for points of twistor space; the tautological section of $\mathscr{O}(1,1)$ over $\mathbf{P}^{*} \times \mathbf{P}$ is denoted by $W \cdot Z$ or by $W_{\alpha} Z^{\alpha}$; for any element $\varepsilon=\varepsilon_{\alpha \beta \gamma \delta} \in \Lambda^{4} \mathbf{T}^{*}$ we write $D X$ for the natural section of $H^{0}\left(\mathbf{P}, \Omega^{3}(4)\right)$ given by

$$
\varepsilon_{\alpha \beta \gamma \delta} X^{\alpha} d X^{\beta} \wedge d X^{\gamma} \wedge d X^{\delta}
$$

and for $\varepsilon=\varepsilon^{\alpha \beta \gamma \delta} \in \Lambda^{4} \mathbf{T}$ we write $D W$ for the section of $H^{0}\left(\mathbf{P}^{*}, \Omega^{3}(4)\right)$ given by

$$
\varepsilon^{\alpha \beta \gamma \delta} W_{\alpha} d W_{\beta} \wedge d W_{\gamma} \wedge d W_{\delta}
$$

The spinor parts of $X$ and $W$ will be denoted by $\left(X^{A}, X_{A^{\prime}}\right)$ and $\left(W_{A}, W^{A^{\prime}}\right)$ respectively; the operation $\rho_{v}$ of restricting a twistor to the projective line $L \subset \mathbf{P}$ or $L^{*} \subset \mathbf{P}^{*}$ corresponding to $v=v^{A A^{\prime}}$ in Minkowski space is given explicitly by

$$
\rho_{v} X=\left(i v^{A A^{\prime}} X_{A^{\prime}}, X_{A^{\prime}}\right) \text { and } \rho_{v} W=\left(W_{A},-i v^{A A^{\prime}} W_{A}\right) .
$$

In this way $\left(X_{A^{\prime}}\right)$ and $\left(W_{A}\right)$ become homogeneous coordinates on $L$ and $L^{*}$ respectively and the natural elements of $H^{0}\left(L, \Omega^{1}(2)\right)$ and $H^{0}\left(L^{*}, \Omega^{1}(2)\right)$ are written as $\Delta X$ and $\Delta W$ respectively. We shall also write

$$
\varpi=\Delta W \wedge \Delta X \wedge \Delta Y \wedge \Delta Z \text {. }
$$

Finally, the squared (complex) Minkowski interval ('distance') between two points $v_{1}$ and $v_{2}$ in the finite part of $\mathbf{M}$ will be denoted by $\left[g\left(v_{1}, v_{2}\right)\right]^{2}$.

4.1. The scalar product for $\mathbf{P} \times \mathbf{P}^{*}$. Here we take $m=2, P_{1}=\mathbf{P}^{*}, P_{2}=\mathbf{P}$, and

$$
h=\frac{D W \wedge D Z}{(W \cdot Z)^{r+4}}
$$

(with $r>-4$ ) so that $h \in H^{6,0}(\Pi-A ; \mathscr{O}(-\mathbf{r}))$. By Theorem 3.2, it is then the homology group $H_{2}(\Lambda-A)$ that gives the functionals on

$$
H^{1}\left(P_{1}-L_{1} ; \mathscr{O}(r)\right) \otimes H^{1}\left(P_{2}-L_{2} ; \mathscr{O}(r)\right)
$$


associated to the kernel $h$. That homology group is quite easy to analyse, however. In terms of the homogeneous coordinates $W_{A}$ on $L_{1}$ and $Z_{A^{\prime}}$ on $L_{2}$, the intersection $\Lambda \cap A$ is given by an equation of the form $t^{A A^{\prime}} W_{A} Z_{A^{\prime}}=0$. Three cases now arise. First, the problem is ill-posed (cf. (16)) if and only if $t^{A A^{\prime}}=0$; this occurs if and only if $v_{1}=v_{2}$.

Secondly, suppose that the $2 \times 2$ matrix $t$ is of rank one; this occurs if and only if $v_{1}$ and $v_{2}$ are distinct but null-separated. In this case, we may change coordinates so that $A$ is given by $W_{0} Z_{0^{\prime}}=0$-then it is clear that $\Lambda-A$ is contractible and there is no functional associated to $h$.

Finally, we suppose that $t^{A A^{\prime}}$ is nonsingular. Then from the Leray exact sequence (9), we find that $H_{2}(\Lambda-A)$ has a unique generator. These calculations precisely reproduce what was already known $[4,5]$.

4.2. The scalar product for $\mathbf{P} \times \mathbf{P}$. Here we take $m=2, P_{1}=\mathbf{P}, P_{2}=\mathbf{P}$, and $\Sigma$ to be the diagonal $\Delta$ in $P_{1} \times P_{2}$. For each $r$, there is a natural holomorphic kernel

$$
h \in H^{6,2}(\Pi-\Delta ; \mathscr{O}(r-2,-r-2))
$$

called by Atiyah [1] the 'Serre class' of $\Delta$ in $P_{1} \times P_{2}$.

Applying Theorem 3.2, we are led to consider the homology group $H_{4}(\Lambda-\Delta)$. Once again, we see that the problem is well-posed if and only if $L_{1}$ and $L_{2}$ are not coincident. Since the real dimension of $\Lambda$ is 4 , our homology group has a generator if and only if $\Lambda-\Delta$ is compact and this is the case if and only if $\Delta$ does not meet $\Lambda$. This in turn happens if and only if $v_{1}$ and $v_{2}$ are not null-separated, just as it should. (We are grateful to A. P. Hodges for suggesting that we include this example.)

4.3. The massless scalar $\phi^{4}$ vertex. Here we take $m=4, P_{1}, P_{3}$ to be copies of $\mathbf{P}^{*}$, and $P_{2}, P_{4}$ to be copies of $\mathbf{P}$. Let

$$
h_{0}=\frac{\Omega}{(W \cdot X)(Y \cdot X)(Y \cdot Z)(W \cdot Z)},
$$

where $\Omega=D W \wedge D X \wedge D Y \wedge D Z$. Then

$$
h_{0} \in H^{12,0}\left(\Pi-\Sigma_{0} ; \mathscr{O}(\mathbf{2})\right)
$$

where $\Sigma_{0}$ is the singularity set of $h_{0}$, and we are interested in the functionals on

$$
H^{1}\left(P_{1}-L_{1} ; \mathscr{O}(-2)\right) \otimes \cdots \otimes H^{1}\left(P_{4}-L_{4} ; \mathscr{O}(-2)\right)
$$

associated to $h_{0}$.

Before continuing, let us point out that for a generic configuration of lines $L_{j}$ one hopes to find three associated functionals corresponding to the three physical channels for the massless $\phi^{4}$ vertex. Moreover, these should have the property that under a continuous motion of the $L_{j}$ into one of the special configurations

(i) $v_{1}=v_{2}$ and $v_{3}=v_{4}$; 
(ii) $v_{1}=v_{4}$ and $v_{2}=v_{3}$;

(iii) $v_{1}=v_{3}$ and $v_{2}=v_{4}$;

precisely one of the three functionals will survive.

However, there are no associated functionals in cases (i) and (ii) since the problem is then ill-posed. In case (iii) (and for a generic configuration of the $v_{j}$ ) the problem is well-posed and we find a single associated functional by considering the group suggested by Theorem 3.2 which is here

$$
H_{4}\left(\Lambda-\Sigma_{0}\right) \text {. }
$$

We omit the details of this calculation since they were written down by Sparling in [16]. Indeed, our general procedure shows that the functionals found by Sparling are the only ones associated to the kernel $h_{0}$. It also confirms a complicated 'bare-hands' calculation of the group (15) which is in this case

$$
H_{16}\left(\left(P_{1}-L_{1}\right) \times \cdots \times\left(P_{4}-L_{4}\right)-\Sigma_{0}\right) .
$$

This involved repeated applications of the Leray exact sequence and the spectral sequence of a fibration; the details appear in [15].

We have just seen that there are no functionals associated, in the sense of $\S 3$, to $h_{0}$ for the special situations of $\Lambda$ corresponding to (i) and (ii). However, in [6] certain functionals at the level of representative cocycles were constructed for all channels (in particular, for the channels corresponding to (i) and (ii)) in such a way as to give the correct (cohomological) answers. How these functionals fit into our general scheme is something of a mystery. The only possibility seems to be that these functionals are represented by products of $h_{0}$ with some other analytic compact cohomology class. This suggestion is to some extent supported by the fact that the procedure employed in [6] fails to respect the freedom inherent in representing cohomology classes by Čech cocycles when it is applied to fields of nonzero spin.

4.4. A new kernel for the $\phi^{4}$ vertex. As a final application of our general method we describe a new kernel $h$ for the $\phi^{4}$ vertex which has the desirable property that there are three functionals associated to $h$ for generic positions of the $L_{j}$ such that one them survives in each of the special configurations (i), (ii), and (iii) of $\S 4.3$. A derivation of this kernel from the corresponding space-time integral is given in Appendix B-a rather deeper reason for its study has emerged in recent work of Hodges [7].

Our kernel $h$ is given by

$$
h=\Omega / \delta^{4},
$$

where

$$
\delta^{2}=(W \cdot X)(Y \cdot Z)-(W \cdot Z)(Y \cdot X),
$$

and we continue to use the notations of $\S 4.3$. This quantity may have a rather unfamiliar appearance: but it is actually very natural, being (essentially) the complexification of the Fubini-Study metric on $\mathbf{P}^{+}$that is associated to the 
(indefinite) Hermitian form $\bar{Z} \cdot Z{ }^{1}$ In fact, the Fubini-Study 'distance' between $X$ and $Z$ in $\mathbf{P}^{+}$is

$$
\frac{\delta(\bar{X}, X, \bar{Z}, Z)}{[(\bar{X} \cdot X)(\bar{Z} \cdot Z)]^{1 / 2}}
$$

Having introduced this kernel, we can apply Theorem 3.2 and are led to study the group

$$
H_{4}(\Lambda-\Sigma) \text {. }
$$

Here we have written $\Sigma$ for the singularity set of $h$ so that

$$
h \in H^{12,0}(\Pi-\Sigma ; \mathscr{O}(\mathbf{2})) \text {. }
$$

Applying the Leray exact sequence to (28) we deduce that its dimension is at most three when the $L_{j}$ are in general position. To show that the generators reflect the structure alluded to at the beginning of this subsection we adopt the following quite explicit approach.

We begin by constructing a generator of (28) for each of the special configurations (i), (ii), and (iii). A routine calculation reveals that the restriction of $\delta^{2}$ to $\Lambda$ is

$$
Q=Q^{A A^{\prime} B B^{\prime}} W_{A} X_{A^{\prime}} Y_{B} Z_{B^{\prime}},
$$

where

$$
Q^{A A^{\prime} B B^{\prime}}=\left(v_{1}-v_{2}\right)^{A A^{\prime}}\left(v_{3}-v_{4}\right)^{B B^{\prime}}+\left(v_{1}-v_{4}\right)^{A B^{\prime}}\left(v_{2}-v_{3}\right)^{B A^{\prime}} .
$$

We now consider the simplifications in this expression that result from the imposition of conditions (i)-(iii).

In case (i), $Q$ reduces to

$$
\left(v_{1}-v_{3}\right)^{A A^{\prime}} W_{A} Z_{A^{\prime}}\left(v_{1}-v_{3}\right)^{B B^{\prime}} Y_{B} X_{B^{\prime}}
$$

where $v_{1}-v_{3}$ must be nonsingular (since $v_{1}$ and $v_{3}$ must not be null separated). Changing the coordinates $\left(W_{A}, Z_{A^{\prime}}\right)$ and $\left(Y_{B}, X_{B^{\prime}}\right)$ if necessary, we may suppose that this nonsingular matrix is the identity; then by setting $W_{A}=\bar{Z}_{A}$ and $Y_{B}=\bar{X}_{B}$ we define a contour $\Gamma_{12,34}$ on which $Q$ is positive and on which

$$
\varpi=\Delta \bar{Z} \wedge \Delta Z \wedge \Delta \bar{X} \wedge \Delta X .
$$

Hence $\int_{\Gamma_{12,34}} \varpi / Q^{2} \neq 0$ and the contour $\Gamma_{12,34}$ represents a nonzero homology class in $H_{4}(\Lambda-\Sigma)$.

Case (ii) is precisely similar to this one and yields a nonzero class $\Gamma_{14,23}$ in $H_{4}(\Lambda-\Sigma)$.

Finally we come to case (iii). Here,

$$
Q^{A A^{\prime} B B^{\prime}}=\frac{1}{2} \varepsilon^{A B} \varepsilon^{A^{\prime} B^{\prime}}\left(v_{1}-v_{2}\right)_{C C^{\prime}}\left(v_{1}-v_{2}\right)^{C C^{\prime}} .
$$

${ }^{1}$ Recall that $\bar{Z} \cdot Z=\bar{Z}_{A} Z^{A}+\bar{Z}^{A^{\prime}} Z_{A^{\prime}}$ and $\mathbf{P}^{+}$is the open subset of $\mathbf{P}$ on which $\bar{Z} \cdot Z>0$. 
Let $t^{A A^{\prime}}$ be Hermitian and positive definite. Define the contour $\Gamma_{13,24}$ by putting

$$
W_{A}=t_{A}{ }^{A^{\prime}} \bar{Y}_{A^{\prime}} \text { and } X_{B^{\prime}}=t_{B^{\prime}}{ }^{B} \bar{Z}_{B} .
$$

Then on $\Gamma_{13,24}$,

$$
Q=\frac{1}{2} g\left(v_{1}, v_{2}\right)^{2} t^{A A^{\prime}} Y_{A} \bar{Y}_{A^{\prime}} t^{B B^{\prime}} \bar{Z}_{B} Z_{B^{\prime}}
$$

is a positive multiple of the nonzero complex number $g\left(v_{1}, v_{2}\right)^{2}$. Hence $\int_{\Gamma_{13,24}} \varpi / Q^{2} \neq 0$ and so $\Gamma_{13,24}$ represents a nonzero class in $H_{4}(\Lambda-\Sigma)$.

Having established that there is a generator of (28) in each of the special configurations, we must show that each of them survives under a continuous deformation as the $v_{j}$ are moved. Under any sufficiently small deformation, the homology classes that we have just defined persist, since the constructions rested on the nonsingularity and positivity of certain matrices. To deal with the general situation, we may use (projective) linear transformations of $\Pi$ to transform the lines $L_{1}, \ldots, L_{4}$ to a configuration which is arbitrarily close to each of the special cases just considered.

\section{Discussion}

There are some interesting open questions suggested by the foregoing.

First, note that we have not yet studied from the point of view of our general procedure any examples other than those described in $\S 4$. In particular it is important to understand under what circumstances the image $l[h \cup \alpha]$ vanishes (cf. (23) and the comment after Theorem 3.2); all that can be said at present is that one will usually be able to check that $l[h \cup \alpha] \neq 0$ by an explicit calculation of the kind given in $\S 4.4$.

A more general question is to relate our general procedure to the methods that have hitherto been used in the subject. For example, although we were led, in $\S 4.3$, to precisely the group that Sparling considered in his investigations of the $\phi^{4}$ vertex, our routes were rather different.

In effect, Sparling uses the composition of four cobord maps (one for each $\left.L_{j}\right)$ to get from a class $c \in H_{4}\left(\Lambda-\Sigma_{0}\right)$ to a class

$$
\hat{c} \in H_{16}\left(\left(P_{1}-L_{1}\right) \times \cdots \times\left(P_{4}-L_{4}\right)-\Sigma_{0}\right)
$$

and then a functional

$$
\omega_{1} \otimes \cdots \otimes \omega_{4} \mapsto \int_{\hat{c}} h_{0} \omega_{1} \cup \cdots \cup \omega_{4}
$$

for $\omega_{j} \in H^{1}\left(P_{j}-L_{j}, \mathscr{O}(-2)\right)$.

Our method, on the other hand, involves the application of one cobord map (defined by the submanifold $\Lambda$ in $\Pi$ ) to get a class

$$
\tilde{c} \in H_{19}\left(\Pi-\Lambda \cup \Sigma_{0}\right)
$$


and then the functional

$$
\omega_{1} \otimes \cdots \otimes \omega_{4} \mapsto \int_{\tilde{c}} h_{0}\left[\omega_{1} \cdots \omega_{4}\right] .
$$

Recent work ${ }^{2}$ has shown that these two functionals are equal. Indeed, if $\left\{\delta_{i}\right\}$ and $\Delta$ denote the cobord maps associated to a pair of submanifolds and to their intersection, respectively, and if $\delta_{*}$ denotes the appropriate Mayer-Vietoris map, then $\Delta \delta_{*}$ is equal to the composition of the $\delta_{i}$. This relation, which can be extended to a larger family of submanifolds, is dual to that between the cup and dot product.

Our general procedure has reduced the problem of seeking functionals associated to a kernel to the calculation of the homology of spaces of the form $\Lambda-\Sigma$. In $\S 4$ we used somewhat ad hoc methods for these calculations; it is clearly very desirable to have some general methods to handle them. We have recently learned from $\mathrm{M}$. G. Eastwood of a promising systematic approach to such calculations which is being explored by Jozsa and Rice. Their method applies when $\Sigma$ is (homotopic to) an algebraic submanifold of $\Lambda$ : their key observation is that one can then use rational algebraic differential forms to calculate $H_{*}(\Lambda-\Sigma)$-that reduces the original topological problem to an algebraic one.

It is a pleasure to acknowledge the interest, encouragement, and assistance of R. J. Baston, T. N. Bailey, E. Dunne, M. G. Eastwood, A. P. Hodges, and R. Penrose in this work. We also thank R. E. Horan for useful discussions during the completion of the manuscript.

\section{APPENDiX A. Differential forms AND Relative COHOMOlOGY}

To use differential forms to represent the relative sheaf cohomology of $\S 2$, we introduce the complex

$$
\bigwedge^{p, q}(X, U ; B)=\bigwedge^{p, q}(X ; B) \oplus \bigwedge^{p, q-1}(U ; B)
$$

(where $\bigwedge^{p, q}(X, B)$ is the space of smooth $B$-valued forms of type $(p, q)$ on $X$ etc.) equipped with the differential

$$
\bar{\partial}_{F}: \bigwedge^{p, q}(X, U ; B) \rightarrow \bigwedge^{p, q+1}(X, U ; B)
$$

defined by the formula

$$
\bar{\partial}_{F}(\alpha, \beta)=\left(\bar{\partial} \alpha, \bar{\partial} \beta+(-1)^{q} \alpha \mid U\right) .
$$

Then the $q$ th cohomology group of this complex is $H^{p, q}(X, U ; B)$ (cf. [2, p. 78] for the corresponding definition of relative cohomology with constant

\footnotetext{
${ }^{2}$ See S. A. Huggett and M. A. Singer, Cohomological contours and cobord maps, Twistor Newsletter (Oxford preprint), no. 30, pp. 29-32, and S. A. Huggett, Cohomology and twistor diagrams, in Twistors in Mathematics and Physics (T. N. Bailey and R. J. Baston, eds.), London Math. Soc. Lecture Notes Series, vol. 156, Cambridge Univ. Press, Cambridge, 1990, for this and other new material.
} 
coefficients). The maps

$$
H^{p, q}(X, U ; B) \rightarrow H^{p, q}(X ; B)
$$

and

$$
H^{p, q-1}(U ; B) \rightarrow H^{p, q}(X, U ; B)
$$

of (1) are respectively induced by $(\alpha, \beta) \mapsto \alpha$ and $\beta \mapsto(0, \beta)$. The fact that $H^{p, q}(X, U ; B)$ is an invariant of the local environment of $F$ in $X$ follows from the fact that a smooth cut-off function with support near $F$ can be used to construct a representative of any class which is supported in any preassigned open neighbourhood of $F$.

The dual theory is constructed from the complex $\bigwedge_{c}^{p, q}(X, U ; B)$ defined by the exactness of the sequence

$$
0 \rightarrow \bigwedge_{c}^{p, q}(U, B) \rightarrow \bigwedge_{c}^{p, q}(X, B) \rightarrow \bigwedge_{c}^{p, q}(X, U ; B) \rightarrow 0,
$$

where the first nontrivial map is extension by zero of compactly supported forms. The $q$ th cohomology group of $\left(\bigwedge_{c}^{p, *}(X, U ; B), \bar{\partial}\right)$ is $H_{c}^{p, q}(X, U ; B)$.

The long exact sequence in cohomology that is induced by the short exact sequence above is just (2); in particular, the connecting homomorphism is induced by $\bar{\partial}$. Once again, any class can be represented by a form supported arbitrarily close to $F$ so these compact relative cohomology groups depend only on $F$ and its immediate neighbourhood in $X$.

In terms of such representatives, (4) is given by

$$
\left(\xi_{1}, \xi_{2}\right) \otimes \eta \mapsto \int_{X}\left(\xi_{1} \wedge \eta+(-1)^{p} \xi_{2} \wedge \bar{\partial} \eta\right),
$$

where $\xi=\left[\xi_{1}, \xi_{2}\right]$.

If $\left[\alpha_{1}, \alpha_{2}\right] \in H_{F}^{p}(X ; B)$ and $\left[\beta_{1}, \beta_{2}\right] \in H_{G}^{q}(Y ; C)$, the cross product $\left[\alpha_{1}, \alpha_{2}\right] \times\left[\beta_{1}, \beta_{2}\right]$ is represented by $\left((\alpha \times \beta)_{1},(\alpha \times \beta)_{2}\right)$, where $(\alpha \times \beta)_{1}=$ $\alpha_{1} \times \beta_{1}$ and

$$
(\alpha \times \beta)_{2}=(1-\tau) \alpha_{1} \times \beta_{2}+(-1)^{q} \tau \alpha_{2} \times \beta_{1}+(-1)^{p} \bar{\partial} \tau \wedge \alpha_{2} \times \beta_{2} .
$$

In these formulae we have written

$$
u \times v=\pi_{X}^{*}(u) \wedge \pi_{Y}^{*}(v)
$$

and in the second of them $\tau$ is a $C^{\infty}$ function on $X \times Y-F \times G$ such that $\tau=0$ in an open neighbourhood of $F \times(Y-G)$ and $\tau=1$ in an open neighbourhood of $(X-F) \times G$. From this, we obtain the promised explicit formula

for the dot product.

$$
\alpha_{0} \cdot \beta_{0}=\bar{\partial} \tau \wedge \alpha_{0} \times \beta_{0}
$$

APPENDIX B. A SPACE-TIME DERIVATION OF THE NEW KERNEL FOR THE $\phi^{4}$ VERTEX

In this appendix we explain what the kernel $h$ of $\S 4.4$ has to do with the first-order massless scalar $\phi^{4}$ vertex. 
First note that if $x$ and $y$ are two points not null-separated in $\mathbf{M}$ then

$$
\frac{\left(W_{A} Y^{A}\right)^{2}\left(X_{A^{\prime}} Z^{A^{\prime}}\right)^{2}}{\left[\delta\left(\rho_{y} W, \rho_{x} X, \rho_{y} Y, \rho_{x} Z\right)\right]^{4}}=\frac{1}{[g(x, y)]^{4}} .
$$

On the spin-bundle over $\mathbf{M} \times \mathbf{M}$, we have

$$
\frac{\Omega}{\delta^{4}}=\frac{\varpi \wedge d^{4} y \wedge d^{4} x}{g^{4}}
$$

because

$$
\Omega=\left(W_{A} Y^{A}\right)^{2}\left(X_{A^{\prime}} Z^{A^{\prime}}\right)^{2} \varpi \wedge d^{4} y \wedge d^{4} x
$$

Therefore, if $f_{1}, \ldots, f_{4}$ are twistor cohomology classes representing the massless fields $\phi_{1}, \ldots, \phi_{4}$ respectively, we have

$$
\begin{aligned}
\int \frac{f_{1}(W) f_{2}(X) f_{3}(Y) f_{4}(Z)}{[\delta(W, X, Y, Z)]^{4}} \Omega \\
\quad=\int_{(x, y)} \frac{\phi_{1}(x) \phi_{2}(y) \phi_{3}(x) \phi_{4}(y)}{[g(x, y)]^{4}} d^{4} y \wedge d^{4} x .
\end{aligned}
$$

It is now sufficient to show that in the physical channel for which $\phi_{1}$ and $\phi_{3}$ are positive frequency and $\phi_{2}$ and $\phi_{4}$ are negative frequency, the right-hand side of (29) is equal to the $\phi^{4}$ vertex

$$
\int \phi_{1}(x) \phi_{2}(x) \phi_{3}(x) \phi_{4}(x) d^{4} x
$$

where the integral extends over real Minkowski space.

Let $u(x)=\phi_{1}(x) \phi_{3}(x)$, let $v(x)=\phi_{2}(x) \phi_{4}(x)$, and let $\tilde{u}(k)$ and $\tilde{v}(k)$ be their respective Fourier transforms. Then by Plancherel's Theorem,

$$
\int u(x) v(x) d^{4} x=\int \tilde{u}(-k) \tilde{v}(k) d^{4} k .
$$

Since $u$ is positive frequency and $v$ is negative frequency, the support of $\tilde{u}$ is contained in the interior $N_{+}$of the future null cone of the origin in momentum space while the support of the integrand on the right-hand side of (30) is contained in the past null cone $N_{-}$of the origin; hence it may be rewritten

$$
\int \tilde{u}(-k) \tilde{v}(k) \chi_{-}(k) d^{4} k,
$$

where $\chi_{-}$is the characteristic function of $N_{-}$. But the Fourier transform of $g(x, 0)^{-4}$ is $\chi_{-}(k)$, and it follows from the convolution theorem that $\tilde{v} \chi_{-}$is the Fourier transform of

$$
\int_{y} \frac{v(y) d^{4} y}{[g(x, y)]^{4}}
$$

Combining this with Plancherel's identity we obtain what we require, that is,

$$
\int_{(x, y)} \frac{u(x) v(y) d^{4} y d^{4} x}{[g(x, y)]^{4}}=\int_{x} u(x) v(x) d^{4} x .
$$




\section{REFERENCES}

1. M. F. Atiyah, Green's functions for self-dual four-manifolds, Adv. Math. Supp. Stud. 7A (1981), 129-158.

2. R. Bott and L. W. Tu, Differential forms in algebraic topology, Graduate Texts in Math., vol. 82, Springer-Verlag, New York, 1982.

3. M. G. Eastwood, R. Penrose and R. O. Wells, Jr., Cohomology and massless fields, Comm. Math. Phys. 78 (1981), 305-351.

4. M. L. Ginsberg, Scattering theory and the geometry of multi-twistor spaces, Trans. Amer. Math. Soc. 276 (1983), 789-815.

5. M. L. Ginsberg and S. A. Huggett, Sheaf cohomology and twistor diagrams, Advances in Twistor Theory (L. P. Hughston and R. S. Ward, eds.), Pitman, San Francisco, Calif., 1979.

6. A. P. Hodges, Twistor diagrams, Phys. A 114 (1982), 157-175.

7. __ Inhomogeneity and crossing symmetry, Twistor Newsletter (Oxford preprint), no. 25, 1987, pp. 19-33.

8. S. A. Huggett, Sheaf cohomology in twistor diagrams, Ph. D. thesis; Oxford, 1980.

9. S A. Huggett and K. P. Tod, An introduction to twistor theory, London Math. Soc. Student Texts, vol. 4, Cambridge Univ. Press, Cambridge, 1985.

10. B. Iversen, Cohomology of sheaves, Springer-Verlag Universitext, Berlin, 1985.

11. H. B. Laufer, On Serre duality and envelopes of holomorphy, Trans. Amer. Math. Soc. 128 (1967), 414-436.

12. J. Leray, Le calcul différentiel et intégral sur une variété analytique complexe (Problème de Cauchy III), Bull. Soc. Math. France 87 (1959), 81-180.

13. R. Penrose and W. Rindler, Spinors and space-time (two vols.), Cambridge Univ. Press, Cambridge, 1986.

14. J.-P. Serre, Un théorème de dualité, Comment. Math. Helv. 29 (1955), 9-26.

15. M. A. Singer and S. A. Huggett, On the homology of the box diagram, in Further Advances in Twistor Theory, Vol. 1 (L. J. Mason and L. P. Hughston, eds.), Longman, Harlow, Essex, UK, and Wiley, New York, 1990.

16. G. A. J. Sparling, Homology and twistor theory, in Quantum Gravity, an Oxford Symposium (C. J. Isham, R. Penrose and D. W. Sciama, eds.), Oxford Univ. Press, Oxford, 1975.

\section{Mathematical Institute, Oxford OX1 3LB, United Kingdom}

Current address (S. A. Huggett): Department of Mathematics and Statistics, Polytechnic South West, Plymouth, Devon PL4 8AA, U.K.

Current address (M. A. Singer): Lincoln College, Oxford OX1 3DR, U.K. 